\title{
Perforated Jejunal Pseudoiverticulum in a Child - A Case Report with Review of Literature
}

\author{
Kavita Krishnan ${ }^{1 *}$ and Wadah Al-Joudi ${ }^{2}$ \\ ${ }^{1}$ Premier Diagnostic Center, Prime Healthcare Group, Dubai, UAE \\ ${ }^{2}$ Prime Hospital, Prime Healthcare Group, Dubai, UAE
}

${ }^{*}$ Corresponding author: Kavita Krishnan, Premier Diagnostic center, Prime Healthcare Group, Dubai, UAE, Tel: +0971559554973; E-mail: drkavita@primehealth.ae

Rec date: February 20, 2018; Acc date: March 26, 2018; Pub date: March 30, 2018

Citation: Krishnan K, Joudi WA (2018) Perforated Jejunal Pseudoiverticulum in a Child - A Case Report with Review of Literature. J Clin Gastroenterol Hepatol Vol.2 No.1:7.

\section{Abstract}

Background: Jejunal diverticula are rare and majority of them are asymptomatic. However rarely, they may present with perforation as an acute abdominal emergency. Hence this condition should be considered in the differential diagnosis and management of small bowel diverticular disease.

Case summary: A 6-year-old child presented with sudden onset of abdominal pain, with vomiting and diarrhea. Clinical Examination showed features of acute abdomen. CT scan showed distended bowel loops with features suggestive of subacute small bowel obstruction. Emergency laparotomy was done, during which jejunal diverticulum with perforation was seen with pus and adhesions. Resection of small segment of bowel with perforated diverticulum and anastomosis was performed. Histopathology showed features of Pseudo diverticulum with perforation. 4 months post-surgery, the child is doing well.

Conclusion: Jejunal diverticula are rare, however perforated diverticula may present as an acute abdominal emergency and could necessitate surgical repair.

Keywords: Jejunal pseudoiverticulum; Abdominal pain; CT scan; Laparotomy

\section{Introduction}

Jejunal diverticula are rare, with an incidence of less than $0.5 \%$ [1] Despite majority of them remaining completely asymptomatic; complications have been reported in $10 \%$ to $30 \%$ of patients [2-4]. These include chronic abdominal pain, diverticulitis, malabsorption, Hemorrhage, obstruction, abscess formation and perforation. Diverticula can be of congenital and acquired type. In congenital diverticula all 3 coats of bowel are present while in acquired or pseudodiverticula muscular layer is absent. Pathogenesis of acquired jejunal diverticula is similar to those in colon: abnormalities in peristalsis, intestinal dyskinesia, and high intraluminal pressures are thought to play a role $[5,6]$. The incidence of jejunal diverticula is very rare in children, we found only three cases reported in literature so far after extensive search [7-9]. We wish to highlight this case in view of its rarity and to consider this entity in the differential diagnosis of acute abdominal pain, in which prompt surgical intervention could be lifesaving.

\section{Case Report}

A 6-year-old child presented to the ER of our hospital with symptoms of acute abdominal pain and Vomiting. The pain started centrally and was later radiating to right iliac fossa. On examination, mildly distended abdomen with guarding in the whole of abdomen was seen. No palpable mass/visceromegaly were noted. Abdominal CT scan showed Fluid and distended small bowel loops seen in the abdomen maximum diameter measuring $2.9 \mathrm{~cm}$. No obvious evidence of bowel wall thickening, a few discrete mesenteric lymph nodes. Plain CT features possibly suggestive of small bowel inflammatory changes/? sub-acute small bowel obstruction (Figure 1).

Diagnostic laparoscopy was done, which showed perforated jejunal diverticulum, followed by Emergency laparotomy. Laparotomy findings: hugely distended small intestine with pus and fluid all over, diverticulum with perforation in the terminal part of jejunum on the antimesenteric border. Multiple mesenteric lymph nodes found, hypervascular appendix. Hence resection of the segment of the small intestine with perforated segment and anastomosis was done. Appendix found hypervascular so appendectomy was done, along with Mesenteric lymph node biopsy (Figure 2).

\section{Gross pathology}

Segment of small bowel measuring $5 \times 4 \times 1 \mathrm{~cm}$ was received, with a diverticulum on anti-mesenteric border measuring $2 \mathrm{~cm}$ in diameter. Area of perforation was noted at the junction of bowel wall and diverticulum. 


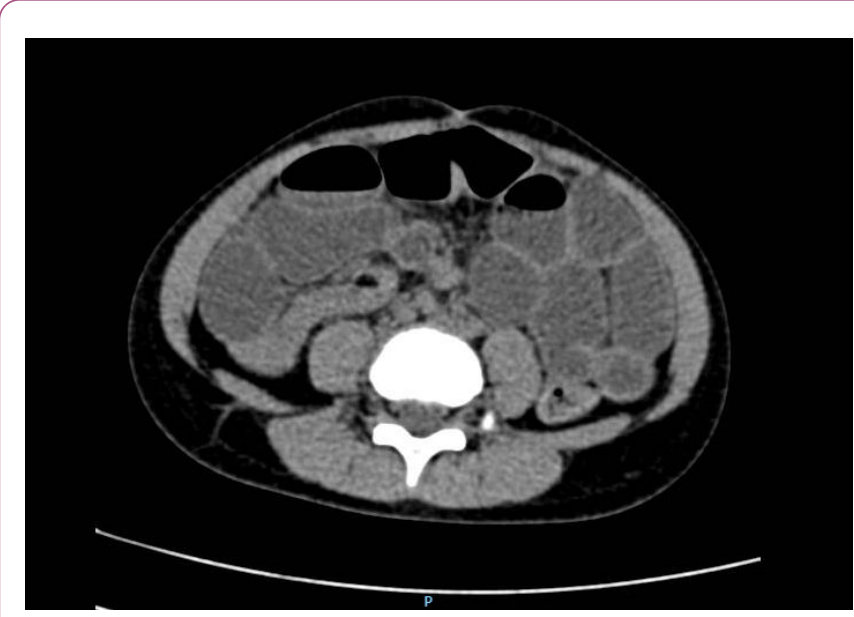

Figure 1 CT scan abdomen - showed fluid and distended small bowel loops - features of subacute intestinal obstruction.

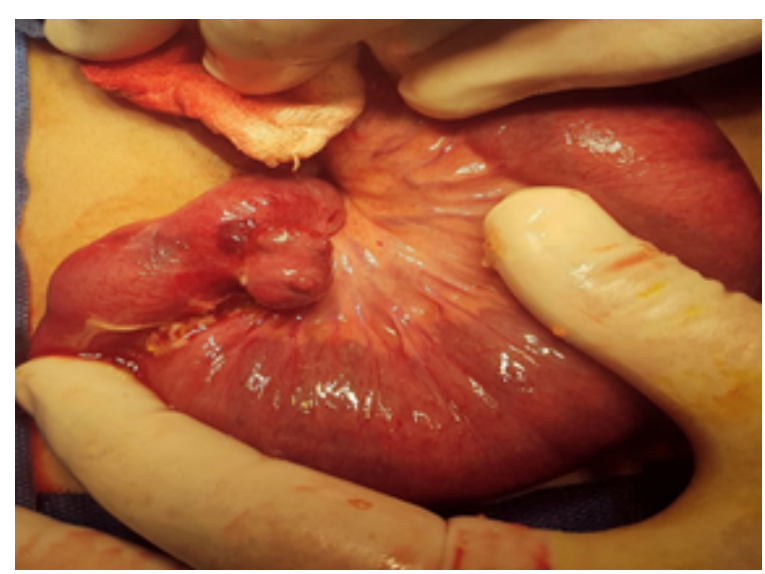

Figure 2 Intraoperative image: Pseudo diverticulum at the terminal part of jejunum, antimesenteric border, with perforation.

\section{Microscopic pathology}

Sections from diverticulum showed small bowel mucosa, with perforation, dense transmural suppurative inflammatory infiltrate and congested vasculature. Muscularis propria was not seen in the wall of the diverticulum. Adjacent small bowel showed inflammation and peritonitis. Appendix showed features of acute appendicitis and mesenteric lymph node showed features of reactive lymphoid hyperplasia (Figure 3).

\section{Discussion}

Jejunal diverticula are the rarest forms of small bowel diverticula, with an incidence of less than $5 \%$ to $0.5 \%$ [1]. The incidence is even rarer in children, with only three cases reported so far on extensive review of literature. Majority of the cases reported so far have been seen in the elderly $[10,11]$. Although the true etiology of this condition is not well known, it is believed to develop from a combination of abnormal Peristalsis, intestinal dyskinesis, and high segmental intraluminal pressures. It is a challenging disorder from a diagnostic perspective. Abdominal imaging may show features of perforation, such as free air under the diaphragm or evidence of obstruction in the form of multiple air fluid levels and bowel dilatation. Endoscopic procedures are useful, however cannot be used in an emergency setting. In the event of obstruction or perforation diagnostic laparoscopy is a useful tool. In the presence of laparoscopic findings such as perforation, abscesses, and mechanical obstruction, exploratory laparotomy is required, resection of the diseased bowel and primary anastomosis is appropriate. Hence considering this condition in the differential diagnosis of acute abdominal emergencies in adults and children with features of intestinal obstruction clinically and on imaging, could alert us in promptly managing the condition and preventing complications such as perforation.

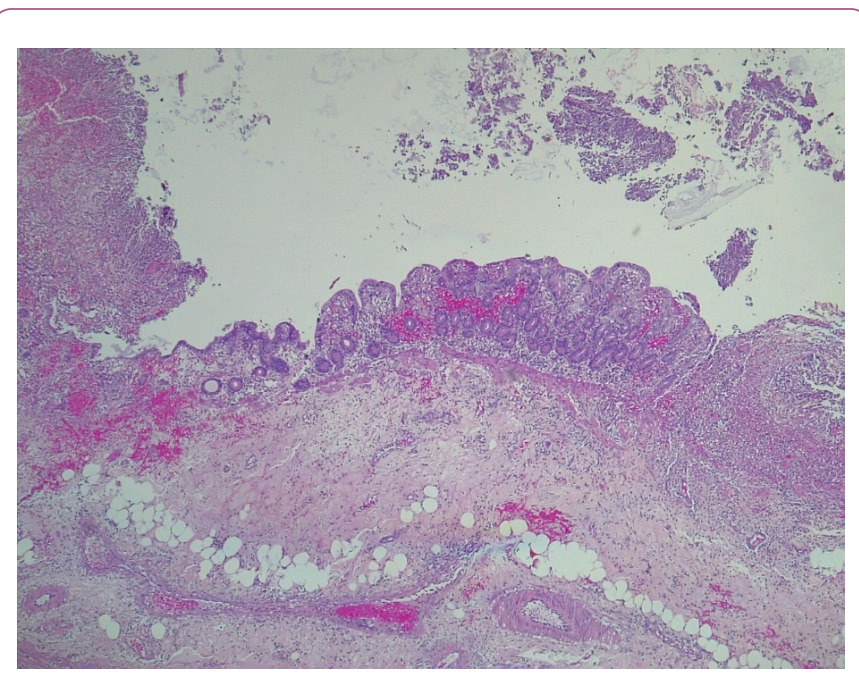

Figure 3 Histopathology of pseudo diverticulum showing mucosa and submucosa without muscularis, features of perforation seen.

\section{Conclusion}

Jejunal diverticulum should be considered in the differential diagnosis of acute or chronic abdominal Symptoms, in view of the high morbidity and mortality associated with delayed diagnosis of the condition and complications such as intestinal perforation, which can be well managed and cured by prompt surgical intervention.

\section{Consent}

Written informed consent was obtained from the patient's family for publication of his medical data.

\section{References}

1. Zager JS, Garbus JE, Shaw JP, Cohen MG, Garber SM (2000) Jejunal diverticulosis: A rare entity with multiple presentations, a series of cases. Dig Surg 17: 643-645. 
2. Wilcox RD, Shatney $\mathrm{CH}$ (1988) Surgical implications of jejuna diverticula. South Med J 81: 1386-1391.

3. Sibille A, Willocx R (1992) Jejunal diverticulitis. Am J Gastroenterol 87: 655-658.

4. Akhrass R, Yaffe MB, Fischer C, Ponsky J, Shuck JM (1997) Smallbowel diverticulosis: Perceptions and reality. J Am Coll Surg 184: 383-388.

5. Gotian A, Katz S (1998) Jejunal diverticulitis with localized perforation and intramesenteric abscess. Am J Gastroenterol 93 : 1173.

6. Maglinte DD, Chernish SM, DeWeese R, Kelvin FM, Brunelle RL (1986) Acquired jejunoileal diverticular disease: Subject review. Radiology 158: 577-580.
7. Cofini M, Quadrozzi F, Cofini G (2015) A perforated jejunal diverticulum in a childhood. J Case Rep 4: 481-483.

8. Sayed L, Mann C, Ihedioha U, Ratliff D (2012) Jejunal diverticulitis in a child. J Surg Case Rep 2012: 9.

9. Bunni J, Barrett HL, Cook TA (2014) Perforated jejunal ulcer associated with gastric mucosa in a jejunal diverticulum. World $\mathrm{J}$ Clin Cases 2: 209.

10. Butler JS, Collins CG, McEntee GP (2010) Perforated jejunal diverticula: A case report. J Med Case Rep 4: 172.

11. Laiz-Díez B, González-González J, Ruiz-Tovar J, López-Monclús J, Durán-Poveda M (2017) Jejunal pseudodiverticulosis. Three cases report. Revista Española de Enfermedades Digestivas 2017: 305-306. 